\title{
A Simple Technique to Maintain Duct Patency- A Technical Note
}

\author{
Dr.R. Appadurai $\mathrm{MDS}^{1}$ Dr.N.Kavitha $\mathrm{MDS}^{2}$, Dr.M.Natarajan $\mathrm{BDS}^{3}$ \\ Dr.D.Lingeshwar $\mathrm{MDS}^{4}$, Dr. R. Manjula BDS ${ }^{5}$ \\ ${ }^{1}$ Associate Professor, Government Royapettah Hospital,KilpaukMedicalCollegeandHospital \\ ${ }^{2}$ Senior Assistant Professor, Government Dharmapuri Medical College and Hospital \\ ${ }^{3}$ Tutor, Government Dharmapuri Medical College and Hospital \\ ${ }^{4}$ Assistant Professor, Government RoyapettahHospital, Kilpauk Medical CollegeandHospital \\ ${ }^{5}$ Senior intern, GovernmentRoyapettahHospital,KilpaukMedicalCollegeandHospital
}

\begin{abstract}
Salivary duct lithiasis is a condition characterized by obstruction of a salivary gland or its excretory duct due to the formation of calcareous concretions or sialolith resulting in salivary ecstasia and even provoking the subsequent dilation of the salivary gland. Sialolithiasis commonly involves the submandibular gland and less frequently the parotid and sublingual gland.We report a case of lithiasis of stenson's duct, its surgical management with modified technique. This modified technique is aimed at maintaining the duct patency using an $18 G$ catheter (IV).
\end{abstract}

Key words: Sialolithiasis, Parotid gland, Stenson's duct, Sialolith

\section{Introduction}

Sialolithiasis is a condition where a calcified mass (sialolith) obstructs the flow of saliva, leading to inflammation of the respective glands (Sialadenitis). Literature shows that sialolith affects more than $\sim 80 \%$ of submandibular ducts and $\sim 15 \%$ of parotid ducts and $\sim 2 \%$ in minor salivary glands. ${ }^{1}$ Sialolith can be formed either in the duct or in the parenchyma of the gland, however sialolith occurring in the duct is more common. Pathophysiology of calculi formation in the duct remains unclear. Various hypothesis have been put forth to understand the possible cause. The basis of these hypothesis conclude, presence of nidus which precipitates deposition of calcareous layers. ${ }^{2,3,4}$ In addition salivary stasis in the duct further contributes to the increasing mass of the calculi. ${ }^{4,5,7}$ Traditional method to remove stones present in the duct is by milking of the duct, in case of larger stones or stones present at the proximal end of the duct a surgical intervention might be required. ${ }^{8}$ The surgical intervention of sialolith removal has increased probability of inducing fistula formation secondary to trauma caused during the procedure. Various techniques have been developed over the years to retrieve the stones while maintaining the patency of duct in case of superficial sialolith. This article describes a case of parotid gland sialolith which was treated using a novel technique, aimed at retrieval of stone, concurrently maintaining duct patency.

\section{Case Report}

A 36 year old female patient reported to dental department complaining of pain at meal time, intraoral swelling and mild pus discharge from upper left $2^{\text {nd }}$ molar region for the past 2 months, not associated with fever. Intraoral examination revealed

a fibrous swelling of the left cheek, not adherent to deeper structuresFig.1. Intraoral periapical radiograph of the region showed a sialolith located in the excretory duct. It was treated with systemic antibiotics and analgesics. CT revealed $1.2 \times 0.8 \mathrm{~cm}$ sized sialolith of left parotid ductFig.2. It was decided to remove the sialolith surgically based on the location and size of the calculus.

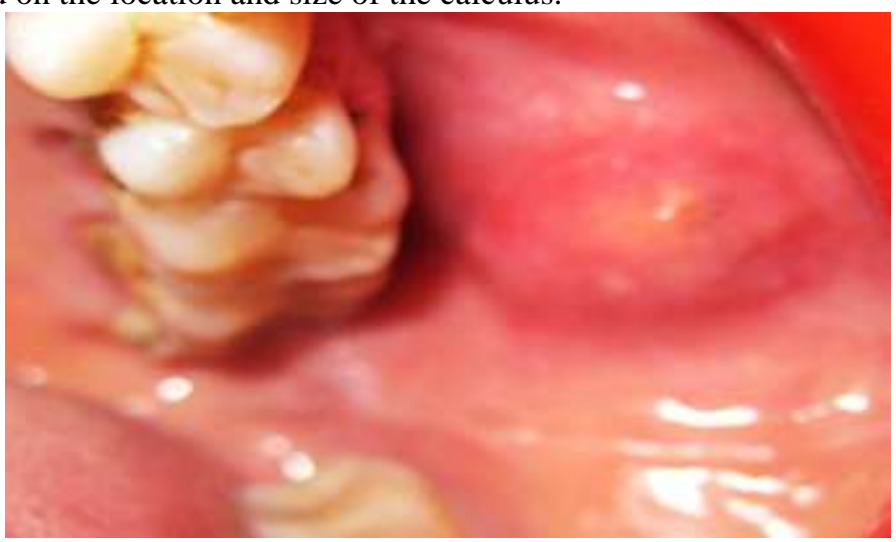

Fig 1- Pre-operative clinical picture revealing a swelling in the left buccal mucosa. 


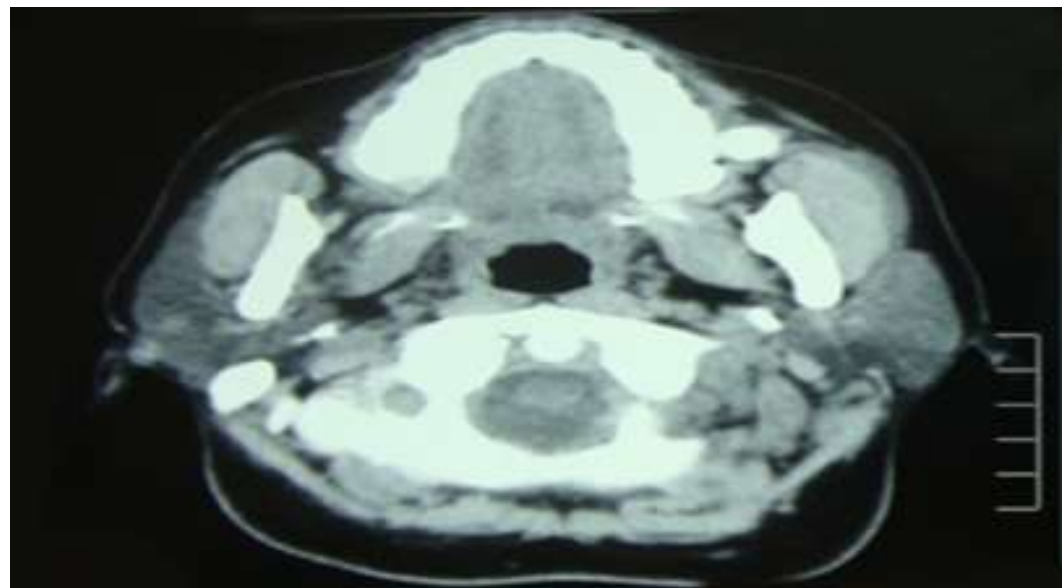

Fig 2 - CT scan report revealing a calcified mass of $1.2 * 0.8 \mathrm{~cm}$ dimension on the left side.

\section{Technique}

- Incision was made over the swelling along the course of the duct and layer wise dissection made to expose the duct with sialolith.

- The patency of the ductal orifice was maintained with an $18 \mathrm{G}$ catheter (IV) to avoid difficulty in catheterizing later.

- Incision was made on the duct exposing the calculi and was delivered through the incisionFig.3

- Once the sialolith was out, the $18 \mathrm{G}$ IV catheter was passed into the distal portion of the incised duct and retained with stay sutures with $3-0$ braided black silk sutureFig.4

- The mucosal incision was closed with $3-0$ braided black silk suture

- Milking of the gland was done and flow of saliva was observed after flushing the catheter with povidone iodine

- To prevent duct collapse by cicatrisation and maintain patency of the duct, the catheter was retained for 7 days post operatively.In successive follow up visits there was complete remission of symptoms, effective salivary drainage and normal functioning of glandFig.5

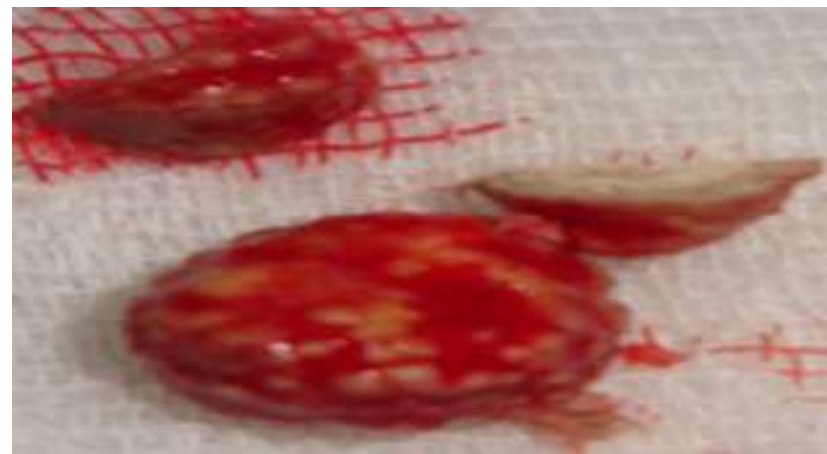

Fig 3 -Clinical picture showing the excised sialolith from the left stenson's duct.

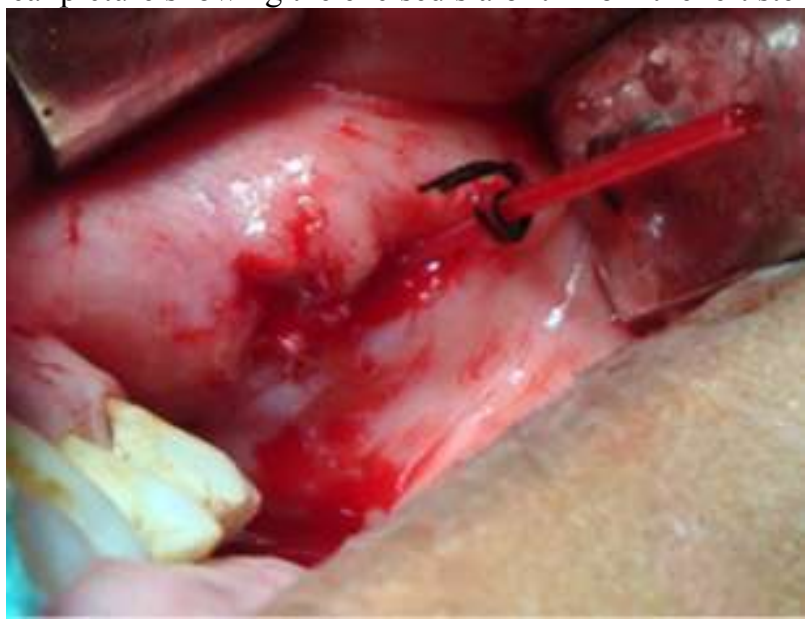

Fig 4 -Clinical picture showing catheterization of the stenson’s duct using an $18 \mathrm{G}$ catheter. 


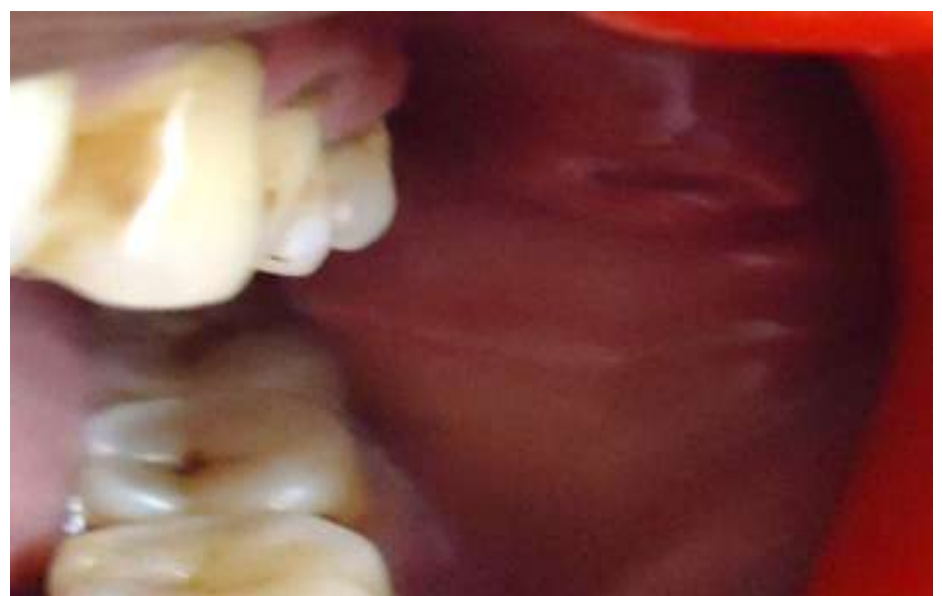

Fig 5 -Post-operative picture showing opening of the ductal orifice being restored after surgical intervention.

\section{Discussion}

Parotid gland is one of the major salivary gland, secretion of which is serous in nature thereby making the occurrence of parotid sialolith a rare one. However when a sialolith is formed in the stenson's duct, depending on its location and size the treatment plan will vary. Sialolith of stenson's duct is often located between hilum of the gland and the point where it takes a turn along the lateral border of the masseter muscle. ${ }^{910}$ In this case sialolith is formed in the distal end of the duct, after it has pierced through the buccinator muscle. Initial line of management of parotid gland sialolith involves conservative approach. Conservative approaches include; warm compresses, massaging the gland, administration of sialologues and antibiotic coverage. ${ }^{11}$ Surgical intervention might be required in case of large or deeply seated sialolith. This case required surgical intervention as the conservative approach did not yield, owing to its size of $1.2 \times 0.8 \mathrm{~cm}$. Various techniques that can be employed for sialolith management are shock wave lithotripsy, sialendoscopy, interventional radiology, endoscopically video-assisted trans-oral and cervical surgical retrieval of stones. ${ }^{12,13,14,15}$ These methods are highly technique sensitive and are not cost effectiveVarious consequences that can occur after surgical intervention include fistula formation and strictures. In case of fistula formation there is new ductal opening. Fistula formation can temporarily aid in infection control, however we may anticipate secondary infection in near future due to modification in the ductal course. ${ }^{10}$ The above discussed technique helps to overcome such complications. In the technique described the duct patency and the viability of the duct canal is maintained. But this technique cannot be employed in case of sialolith occurring in the proximal part of the duct. In such cases sialadenectomy or total conservative parotidectomy can be employed. ${ }^{8,15}$

\section{Acknowledgement and Disclosure}

1. We would like to acknowledge the Anesthetic team of Government Dharmapuri Medical College.

2. "The authors report no conflicts of interest related to this case report", or "No external funding, apart from the support of the authors' institution, was available for this case report".

\section{References}

[1]. Bodner L. Salivary gland calculi: Diagnostic imaging and surgical management. Compendium, 1993;14:572-86.

[2]. Bernier, J. L.: The Management of Oral Disease, St. Louis, 1959, The C. V. Mosby Company.

[3]. Thoma, K. H.: The Use of Radiopaque Media in Roentgen Diagnosis of Oral Surgical Conditions, Am. J. Orthodontics \& Oral Surg, 27: 64-69, 1941

[4]. Archer, W. H.: Manual of Oral Surgery, Philadelphia, 1952, W. B. Saunders Company.

[5]. Levy, D. M., ReMine, W. H., and Devine, K. D.: Salivary Gland Calculi, J.A.M.A, 181: 11151121, 1962

[6]. Erickson, R. I., and Hale, M. L.: Minor Salivary Gland Sialolithiasis; Report of a Case, Oral Surg Oral Med Oral Pathol Oral Radiol Endod 15: 200-203, 1962.

[7]. Moskow, B. S., and Robinson, H. L.: Minor Salivary Gland Sialolithiasis; Report of a Case, Oral Surg Oral Med Oral Pathol Oral Radiol Endod 17: 225-227,1964

[8]. Hald J, Andreassen UK. Submandibular gland excision: short- and long-term complications. ORL J Otorhinolaryngol Relat Spec 1994;56:87-91.

[9]. Scheman, P.: New Surgical Approach to Treatment of Recurrent Sialolithiasis of Sub-maxillary Gland, New York State D. J, 23: 355-359, 1957.

[10]. Williams MF.Sialolithiasis. Otolaryngol Clin North Am 1999;32:819-34.

[11]. Iro H, Nitsche N, Schneider TH, Ell C. Extracorporeal shockwave lithotripsy of salivary gland stones. Lancet 1989; 2:115.

[12]. Marchal F, Becker M, Dulguerov PO, Lehmann W. Interventional sialendoscopy. Laryngoscope 2000; 110:318-20.

[13]. Drage N, Brown JE, Escudier M, McGurk M. Interventional radiology in the removal of salivary calculi. Radiology 2000;214:13942.

[14]. Nahlieli O, London D, Zagury A, Eliav E. Combined approach to impacted parotid stones. J Oral Maxillofac Surg 2002;60:1418-23.

[15]. Beahrs OH. The facial nerve in parotid surgery. Surg Clin North Am 1963; 43:973-7. 